\title{
Learning Motor Synergies Makes Use of Information on Muscular Load
}

\author{
Juan Fernández-Ruiz, ${ }^{1,3}$ Cynthia Hall-Haro, ${ }^{1}$ Rosalinda Díaz, ${ }^{1}$ Jacqueline Mischner, ${ }^{1}$ \\ Patricia Vergara, ${ }^{1}$ and Juan Carlos Lopez-Garcia ${ }^{2}$ \\ ${ }^{1}$ Laboratorio de Neuropsicología, Departamento de Fisiología, Facultad de Medicina, Universidad Nacional Autónoma de México, 04510 México \\ D.F., México; ${ }^{2}$ Instituto Cajal Madrid 28002, Spain
}

\begin{abstract}
Prism adaptation, a form of procedural learning, requires the integration of visual and motor information for its proper acquisition. Although the role of the visual feedback has begun to be understood, the nature of the motor information necessary for the development of the adaptation remains unknown. In this work we have tested the idea that modifying the arm load at different stages of the adaptation process, and the ensuing change of motor information perceived by the subjects, would modify the final properties of the adaptation. We trained a set of subjects to throw balls to a target while wearing prism glasses and varied the weight of their arms at different time points during the task. We observed that the acquisition of the adaptation was not affected by the change in load. However, its persistence (i.e., the aftereffect) was reduced when tested under a weight condition different from the training trials. Furthermore, when the training weight conditions were restored later during testing, a second, late aftereffect was unmasked, suggesting that the missing aftereffect did not disappear but had remained latent. Our results show that the internal representation of a motor memory incorporates information about load conditions and that the memory stored under a specific weight condition can be fully retrieved only when the original training condition is restored.
\end{abstract}

The proper completion of a visually guided motor behavior requires neural transformations that convert visual and motor inputs into precise motor outputs. Despite the complexity of the brain activity that underlies it, hand-eye co-ordination in humans is extraordinarily flexible, as illustrated by the ease with which throwing can be adapted to distortions in visual perception. Prism adaptation, a form of procedural learning, is a paramount example of this plastic capability (Harris 1965; Welch 1974; Kornheiser 1976). During this task, the motor system adapts gradually to new visuospatial co-ordinates imposed by prisms that displace the visual field, giving rise to a horizontal shift between the direction of gaze and the direction of movement (Martin et al. 1996). Once the prisms are withdrawn, the strength of the adaptation can be measured by the aftereffect, the spatial deviation of the motor actions in the direction opposite to the visual displacement imposed by the prisms (Fernández-Ruiz and Díaz 1999).

Despite the existence of a large body of psychophysical work, a clear understanding of the kind of information acquired and stored by the nervous system during prism adaptation has only begun to emerge (Cohen 1966; Held et al. 1966; Hardt et al. 1971; Welch et al. 1974; Jakobson and Goodale 1989). Thus, it has been shown that visual feedback is a relevant factor for the onset of the adaptation

${ }^{3}$ Corresponding author.

E-MAIL jfr@servidor.unam.mx; FAX 525-623-2241. because introducing a delay between the movement itself and the visual perception of its result reduces the speed at which the accommodation occurs (Kitazawa et al. 1995). On the other hand, the exact nature of the motor information necessary for the development of the adaptation remains to be determined. One approach to address this issue was used recently by Kitazawa et al. (1997). By using a reaching task in which the subjects were required to respond at various speeds, these authors obtained evidence that arm velocity is an important source of information during prism adaptation, because learning to point to the actual position of the target did not generalize across the different velocities tested.

There are two other sources of information that could play a role in the adaptation process. One is muscular proprioception. When throwing a ball, the information about the length and tension of the muscles, the angles of the joints, and the position of the body in space is conveyed to the central nervous system by at least two kinds of specialized muscle and joint proprioceptors: the muscle spindles, receptors that respond to stretch in specialized muscle fibers, and the Golgi tendon organs, receptors sensitive to changes in tension (Houk and Henneman 1967; Ghez 1991). The other possible source of information is the corollary discharge (Sperry 1950), also known as efference copy of the motor command (von Holst and Mittelstaedt 1950), which has been proposed as a mechanism by which the nervous system can know about its own motor activity (Jeannerod 1995).

LEARNING \& MEMORY 7:193-198 @ 2000 by Cold Spring Harbor Laboratory Press ISSN1072-0502/00 \$5.00

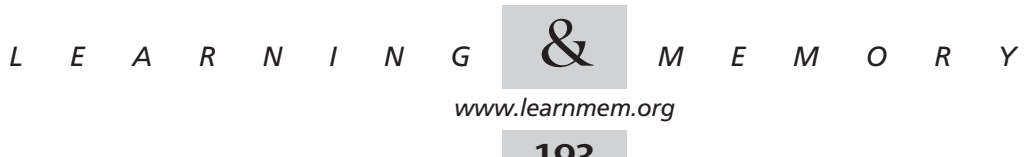


In this work we have explored the possibility that arm load affects prism adaptation as well as the magnitude and the persistence of the aftereffect. To this end, we have trained a set of healthy subjects to throw balls at a target while wearing prism glasses and varied the weight of their arms with wrist bracelets. Our results indicate that the internal representation of a motor memory incorporates information about muscle tension and/or the efference copy and that the memory (i.e., the aftereffect) stored under a specific weight condition can be fully retrieved only when the original training condition is restored.

\section{RESULTS}

\section{Changes in Arm Load Do Not Affect the Initial Throwing Performance in the Horizontal Axis nor the Onset of the Adaptation}

The simple act of throwing clay balls to the target before the subjects donned prisms (PRE) was not affected by the switch from the no-weight to weight condition. As can be seen in Figures 2 and 3, below, the horizontal accuracy of the throws was homogenous throughout all the PRE trials, and no differences were observed between the two throws at the weight transition, revealing a rapid rectification of the ballistic throwing movement. A two-way repeated measures ANOVA conducted on all PRE throws in the four experimental groups confirmed that there were no statistically significant differences in the horizontal axis between the throws made without bracelets and those made with the weights. Regarding the vertical axis, there was a significant difference (paired Student's $t$ test, $P<0.05$ ) between the throws made with and without the weights during the PRE phase. All of the PRI (the subjects having donned the prism glasses) trials in the experimental groups were performed by the subjects wearing weights on their wrists. As can be seen in Figures 2 and 3, below, prism adaptation in these groups proceeded at a similar rate when compared with the control subjects that wore no bracelets. The PRI data were best fitted by a reciprocal quadratic function of the form $f=1 / a+b x+c x^{2}(r=0.99)$. To obtain the rate of change the following derivative function was used: $\mathrm{f}^{\prime}=-\mathrm{b}+2 \mathrm{cx} /$ $\left(a+b x+c x^{2}\right)^{2}$. In order to compare the rate of change for the different groups during the PRI trials, a Kruskal-Wallis test was done. The analysis of the derivatives did not show any differences between any of the groups during the adaptation. Finally, a one-way ANOVA showed no significant differences in the magnitude of the deviation observed on the first PRI trial among the different groups regardless of the weight or in the adaptation value (see Materials and Methods) across all the conditions tested. Thus, the addition of weight to the throwing arm, within the limits we have explored in this study, is not capable of modifying the development of the motor adaptation. Contrary to the adaptation showed in the horizontal axis, the throws made dur- ing the PRI phase remained constant when measured in the vertical axis, as suggested by a repeated measures ANOVA analysis.

\section{The Retrieval of the Motor Memory Is Dependent on the Arm Load Registered During Learning}

Once the subjects had adapted to the prisms, these were removed, and we proceeded to analyze the aftereffect in two different ways. In the first case, the subjects started the POS trials free of the bracelets and halfway through the session switched back to carrying the load. In the second, the mirror image of the first, the subjects started the POS throws with the extra weight, and the bracelets were removed halfway through the trial (Figure 1).

Subjects that performed the initial POS (having removed the prisms) throws without weights (Fig. 1, groups 4 and 5), exhibited a significant reduction in the aftereffect when compared with the control group (group 1) (one-way ANOVA, $F(2,27)=4.87, P=0.01)$. As can be seen in Figures 2 and $4 \mathrm{~A}$, there were reductions of $33.6 \%$ and $43.9 \%$ in the magnitude of the deviation on the first POS throw in the 750-gram and in the 1500-gram groups, respectively, compared with the control subjects. Furthermore, the total sum of the distances between the impact of the ball and the target for the first half of the POS throws (i.e., the initial aftereffect persistence) was also significantly reduced for the 1500-gram group and slightly decreased for the 750gram group, although it did not reach statistical levels of significance in the latter case (Fig. 4B, below). Thus, a reduction in the weight of the arm between training and testing decreases the magnitude of the aftereffect, suggesting that information on arm load is one of the elements encoded into the internal representation of the adaptation.
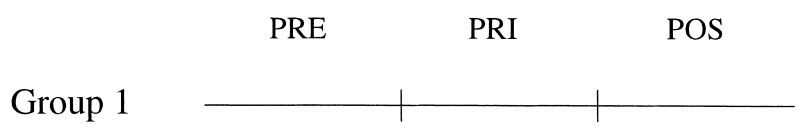

Group 2

Group 3

Group 4

Group 5

Figure 1 Experimental design. The thicker lines represent the period during which weights were used to change the muscular tension of the arm. Groups 2 and 4 wore 750-gram bracelets, whereas groups 3 and 5 wore 1500-gram bracelets. (PRE) Throws before donning 30-diopter lenses; (PRI) throws with the lenses on; (POS) throws after removing the lenses.

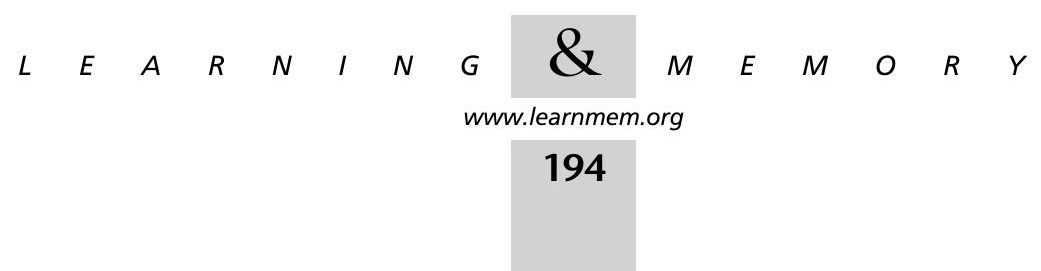




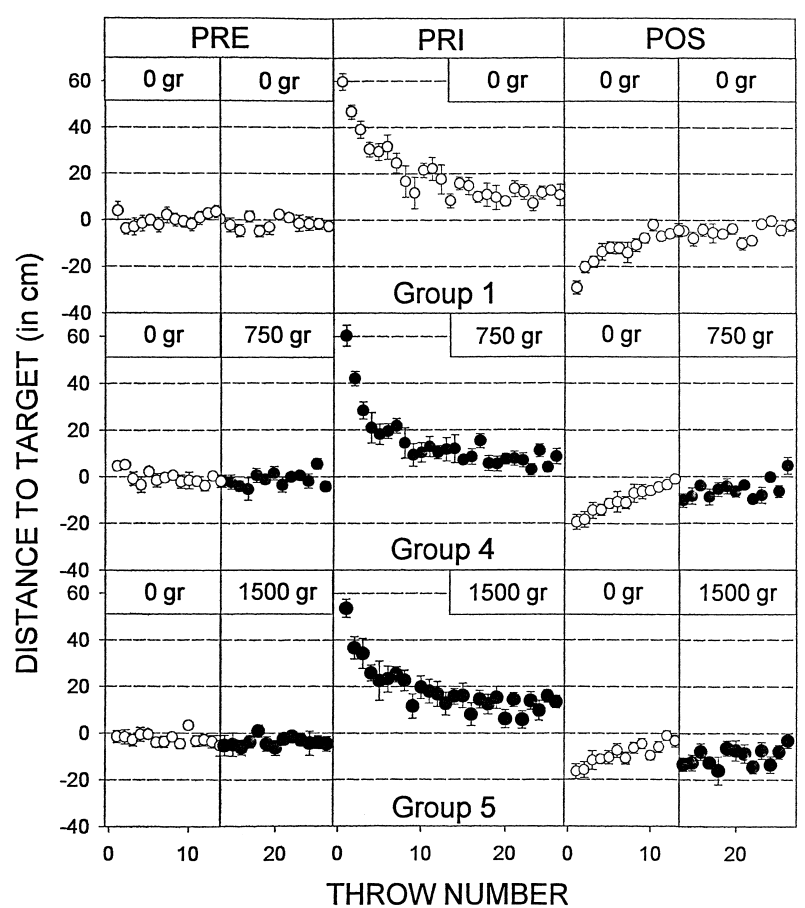

Figure 2 Prism adaptation and aftereffect in groups 1,4 , and 5 . Plots of trial number vs. horizontal displacement from the target for throws made before, while, and after the subjects donned 30-diopter prism lenses. The addition of weight to the throwing arm (groups 4 and 5) during the PRE and PRI trials had no effect on the performance of the task or on the onset of the adaptation. However, the weight-no weight transition between PRI and POS was associated with a smaller aftereffect. Furthermore, the reinstatement of the load halfway through the POS trials led to the appearance of a second, late aftereffect in both experimental groups. Data represent means \pm S.E.M.

Halfway through the POS trials, bracelets were placed on the wrist of these same subjects (groups 4 and 5) such that the muscular tension in their throwing arm was the same as during the training trials. Figure 2 shows that the aftereffect exhibited by both experimental groups had already disappeared before the no-weight to weight transition (i.e., the memory was already extinguished). Nevertheless, the addition of the load elicited a second, late aftereffect in both groups that tended to disappear with further throws (Fig. 2), and its actual magnitude recorded in the fourteenth POS trial (first trial with bracelets) is shown in Figure 4A. A direct within-group comparison between the deviations observed in the thirteenth and fourteenth POS throws (Fig. 5, below) revealed a highly significant difference in the 750gram and the 1500-gram groups (two-way repeated measures ANOVA, $F(2,57)=3.48, P<0.05$ ), providing compelling evidence of the existence of the late aftereffect. Finally, the late aftereffect persistence was also significantly increased for the 1500-gram group and slightly larger for the 750-gram group, although it failed to reach statistical levels of significance (Fig. 4B). The appearance of this second deviation in the accuracy of the throws stands in sharp contrast to the observation made on the PRE trials where the change in tension caused by the addition of the bracelets was rapidly compensated by the motor system. Interestingly, the reduction in the initial aftereffect observed in these subjects (Figs. 2 and 4A,B) can, in principle, be accounted for by the magnitude of the late aftereffect. The sum of the two aftereffects was remarkably homogeneous (control, $33.78 \mathrm{~cm}$; group 4, 29.64; group 5, 29.8), and no statistically significant differences were observed among the groups.

One prediction derived from the appearance of a late shift in the throwing accuracy is that if the second deviation is a real aftereffect revealed by the re-establishment of the original training conditions, then extinguishing the original aftereffect under constant tension should not be followed by a late aftereffect after a weight to no-weight transition. Figure 3 shows that this is the case. Groups 2 and 3 continued wearing bracelets during the initial 13 POS throws (Fig. 1). When compared with the control subjects, no differences were observed in the magnitude of the deviation on the first POS throw (Figs. 3 and 4A) nor in the initial

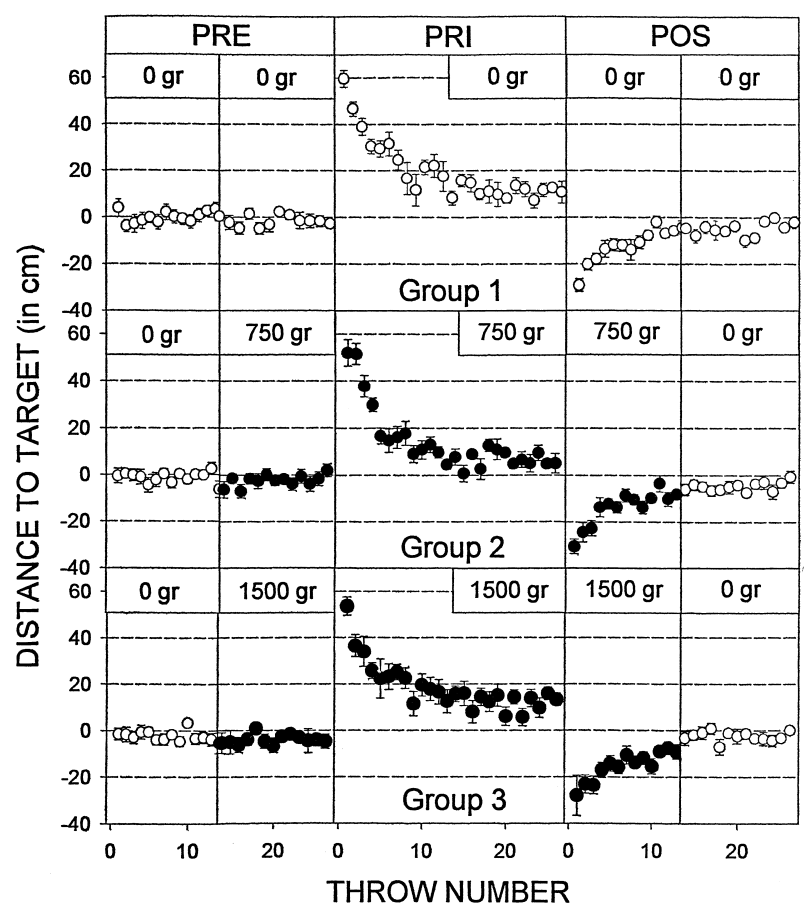

Figure 3 Prism adaptation and aftereffect in groups 1,2 , and 3 . Plots of trial number vs. horizontal displacement from the target for throws made before, while, and after the subjects donned 30-diopter prism lenses. The addition of weight to the throwing arm (groups 2 and 3) had no effect on the performance of the task, the onset of the adaptation, or the magnitude of the aftereffect. Similarly, the removal of the load halfway through the POS trials did not reveal any latent aftereffect in either group. Data represent means \pm S.E.M.

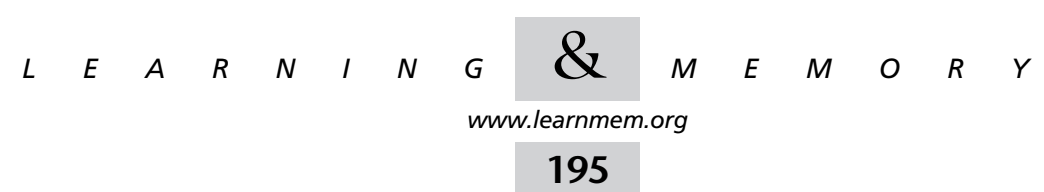



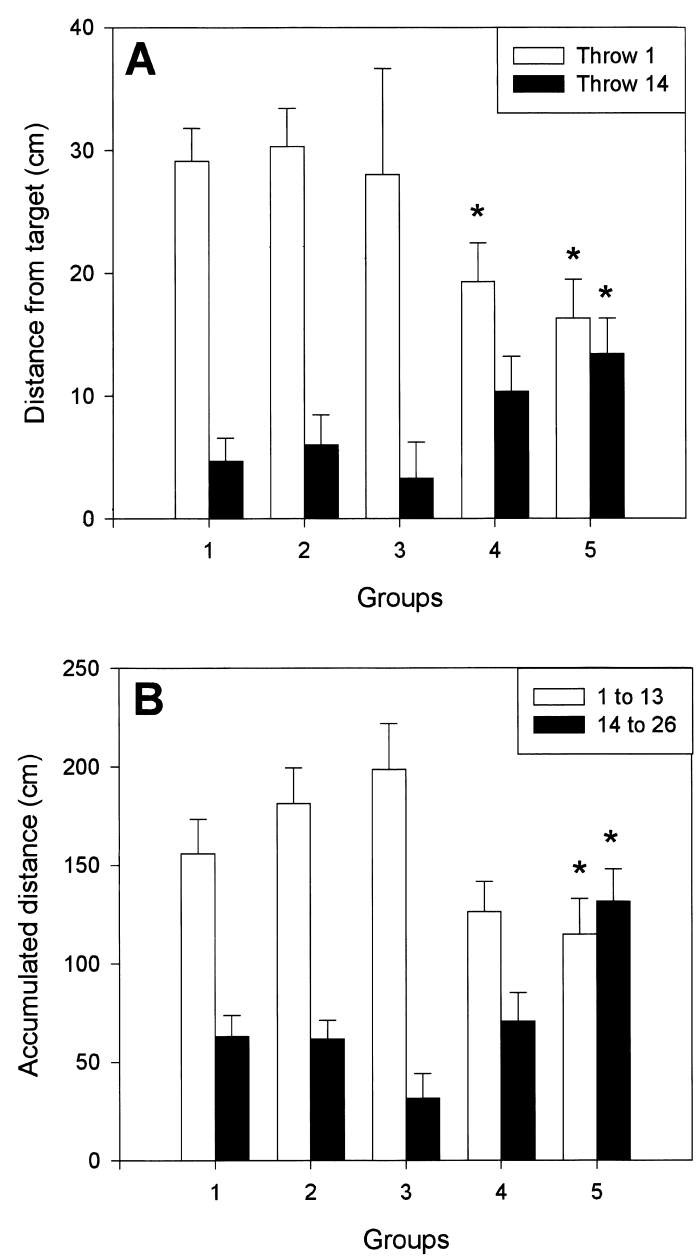

Figure 4 (A) Magnitude of the aftereffect (distance from the target in centimeters) at the beginning of, and halfway through, the POS trials. Groups 4 and 5 exhibited a smaller aftereffect on the first POS throw (light bars) as a result of the load-no load shift. The reinstatement of the load at the fourteenth throw (dark bars) was accompanied by a second aftereffect. In contrast, load transitions in groups 2 and 3 had no effect. (B) Similar results were observed for the aftereffect persistence (accumulated distance from the target in centimeters). $\left.{ }^{*}\right) P<0.05$ as compared with the control group. Data represent means \pm S.E.M.

aftereffect persistence (Fig. 4B). Halfway through the trial, the weights were removed, and no deviation was observed between the first load-free throw and the previous one (Figs. 3 and 4A). Furthermore, the late aftereffect persistence was also homogeneous across the groups (Fig. 4B), and no within-group differences were observed between the deviation recorded in the thirteenth and fourteenth POS throws (Fig. 5). Together, these data indicate that the adaptation had fully disappeared by the time the weight was removed and suggest that the second deviation observed in groups 4 and 5 is a true aftereffect.

Regarding the changes in the vertical axis, the analysis suggested that each time the weights were added, the

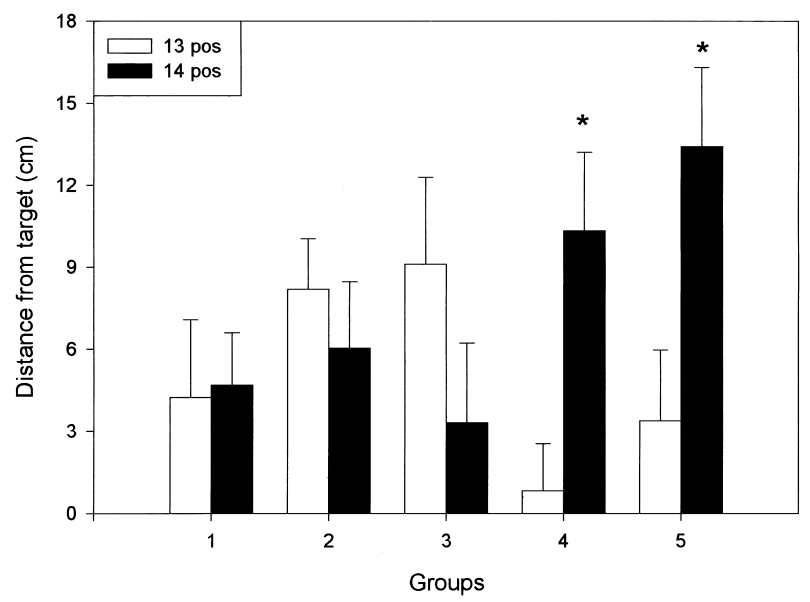

Figure 5 Direct comparison between the horizontal displacements recorded for the two throws at the load transitions (throw 13, light bars vs. throw 14, dark bars) during the POS trials. Significant differences (i.e., a late aftereffect) were observed only for cases in groups 4 and 5 where the reintroduction of the weight re-established the conditions under which the initial adaptation occurred. $\left.{ }^{*}\right) P<0.05$ vs. the corresponding thirteenth throw. Data represent means \pm S.E.M.

throws were shifted downwards, and when the weights were removed, the throws went back up (paired Student's $t$-tests, $P<0.05$ for all cases). In contrast to the displacements observed within each phase in the horizontal axis, there were no in-phase displacements observed when the measurements were done in the vertical axis.

\section{DISCUSSION}

In the present study we have investigated whether proprioceptive information about muscular load plays a role in the acquisition and retrieval of a motor memory, in a prism adaptation task. We explored this issue by asking whether the adaptation of a movement acquired under a specific weight condition could be transferred to a movement of equal amplitude and direction but performed under a different arm load. We found that there was a certain degree of transfer evidenced by the aftereffect observed in the subjects trained while wearing bracelets but tested initially in the load-free condition (groups 4 and 5). This transfer was incomplete because the aftereffect magnitude was significantly smaller in these subjects as compared with the control group. Interestingly, the motor memory that was not transferred did not simply disappear; instead, it remained latent and could be elicited by restoring the weight conditions experienced during training. This manipulation disclosed a second aftereffect of a magnitude equivalent to the reduction observed in the initial deviation.

Two lines of evidence indicate that the late aftereffect is the expression of a dormant motor memory and not an artifact derived from the sudden change in arm load. First, the switch from weight to no-weight during the PRE trials

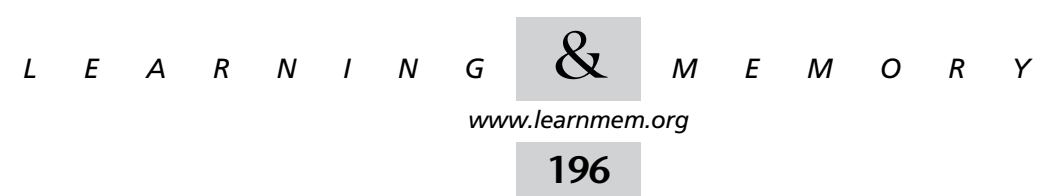


was not accompanied by any deviation on the accuracy of the throws, showing that the motor system is capable of an instantaneous correction of the movement needed to accomplish the task successfully. Second, when the motor memory had been extinguished under load conditions that matched the training protocol, no deviation was observed after the weight to no-weight transition, illustrating once more that an abrupt change in tension within the limits we tested is not enough to modify the precision of the throw.

Our findings demonstrate that the reinstatement of the aftereffect observed in this experiment represents the retrieval of a motor memory that can be elicited only under the conditions similar to when it was acquired. Thus, the internal representation of a motor synergy (i.e., the adaptation) does incorporate information on muscular load. The information could be either proprioceptive or an internal feedback, like the corollary discharge of the motor command. These results are in agreement with previous findings by Gandolfo et al. (1996), which showed that the motor control system can build an internal model of external forces in intrinsic (muscles and joints) co-ordinates. Furthermore, the fact that the motor memory (i.e., the aftereffect) stored under a particular muscle load can be fully retrieved only after the training condition is restored argues that this information is not unspecific but is at least partly restricted to the specific state of the set of muscles during the adaptation process.

In conclusion, our results suggest that the nervous system uses muscular load information when learning motor synergies and that the stored information can be fully retrieved when the original conditions are matched subsequently. In this sense, these findings are in accordance with the proposal by Shadmehr and Mussa-Ivaldi (1994) and Gandolfo et al. (1996) who have suggested that the motor system actively uses muscle information and that learning that occurs under a given set of circumstances remains private to the conditions in which it was originally acquired. Finally, the present results, together with previous findings (Kitazawa et al. 1997; Krakauer et al. 1999), indicate that the perceptuomotor learning of the kind acquired during prism adaptation is not a single phenomenon, but it involves at least two different processes that take place simultaneously and independently for the expression of a final learned motor behavior.

\section{MATERIALS AND METHODS}

\section{Subjects}

Fifty unpaid, right-handed volunteers between the ages of 18 and 24 participated in this study. All subjects were healthy at the time of the experiment and had no history of neurological injury. The subjects were naive to the purpose of the experiment and gave informed consent to participate before the experiments in accordance with the Declaration of Helsinki.

\section{Throwing Procedure}

We followed the throwing technique we had used previously (Martin et al. 1996; Fernández-Ruiz and Díaz 1999). The subjects threw clay balls (weight, 10 grams) to a $12 \times 12-\mathrm{cm}$ cross drawn on a large sheet of parcel paper centred at shoulder level and placed $2 \mathrm{~m}$ away from them. The subjects were instructed to make each toss overhand during the whole experiment and were asked to throw the balls to the location where they saw the target. The subjects performed the task from a standing position and had an unobstructed view of the target during the entire session. The head was unrestrained, and no directions were given about trunk, shoulder, or head/neck posture. However, they were not allowed to look down at their hand as they collected the next ball from a tray located right next to their bodies.

A baseline throwing performance was obtained by asking the subjects to simply throw 26 balls to the cross (condition PRE). The position at which the balls made an impact on or around the target was marked immediately after each throw. At the end of this stage, the volunteers viewed the target binocularly through 30-diopter Fresnel 3M Press-on plastic lenses (3M Health Care, Specialities Division, St. Paul, MN) and were instructed to throw 26 more balls with the same arm and in the same way as before (condition PRI). The prisms were then removed, and the subjects threw 26 more balls (condition POS). During these two conditions, PRI and POS, the subjects could use their knowledge of the deviation of their throws to correct the initial error caused by donning and doffing the prisms. The locations of the impacts were plotted sequentially by trial number versus horizontal and vertical displacement (in centimeters) from a vertical line passing through the center of the target. Impacts to the left or below were plotted as negative values and impacts to the right or above as positive values. It is noteworthy that the prisms produced only a horizontal gaze displacement. Vision along the vertical axes remained unaffected throughout the experiment.

\section{Variations in Arm Load}

To modify the arm load during the throwing task, the subjects wore wrist weight bracelets during different phases of the experiment. Subjects were divided into five groups (five men and five women in each group), and the following experimental design was used (Fig. 1): group 1 (control), no bracelets throughout the experiment; group 2, 750-gram bracelets between the fourteenth PRE throw and the thirteenth POS throw; group 3, 1500-gram bracelets between the fourteenth PRE throw and the thirteenth POS throw; group 4, 750-gram bracelets between the fourteenth PRE throw and the twenty-sixth PRI throw and between the fourteenth and the twenty-sixth POS throws; group 5, 1500-gram bracelets between the fourteenth PRE throw and the twenty-sixth PRI throw and between the fourteenth and the twenty-sixth POS throws.

\section{Magnitude and Persistence of the Adaptation}

Besides obtaining the measurement of all the PRE, PRI, and POS throw impacts, three additional measures were calculated from the collected data. First, the adaptation value was defined as the distance between the impact of the ball and the center of the cross on the final PRI throw minus the distance on the initial PRI throw. Second, the aftereffect magnitude was defined as the distance from the impact to the cross on the first and the fourteenth throws after removing the prisms (initial and late aftereffect, respectively). Third, the aftereffect persistence was defined as the total sum of the deviations for the impacts from the first to thirteenth throws

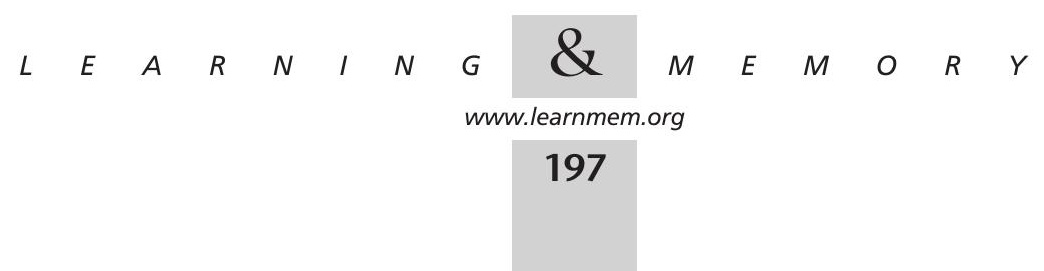


(initial persistence) and from the fourteenth to twenty-sixth throws (late persistence).

\section{Statistical Analysis}

To analyze the effect of wearing weights during the throws, a two-way repeated measures ANOVA was conducted on all PRE throws from the four experimental groups. The learning rate during the PRI condition was analyzed fitting the data to a curve and then obtaining the derivative. After the derivatives were obtained, a Kruskal-Wallis test was used to make the statistical comparisons.

The publication costs of this article were defrayed in part by payment of page charges. This article must therefore be hereby marked "advertisement" in accordance with 18 USC section 1734 solely to indicate this fact.

\section{REFERENCES}

Cohen, H.B. 1966. Some critical factors in prism adaptation. Am. J. Psychol. 79: 285-290.

Fernández-Ruiz, J., and Díaz, R. 1999. Prism adaptation and aftereffect Specifying the properties of a procedural memory system. Learn. E Mem. 6: 47-53.

Gandolfo, F., Mussa-Ivaldi, F.A., and Bizzi, E. 1996. Motor learning by field approximation. Proc. Natl. Acad. Sci.. 93: 3843-3846.

Ghez, C. 1991. The control of movement. In Principles of neural science, 3rd ed. (eds. E.R. Kandel, J.H. Schwartz, and T.M. Jessell), pp. 533-547. Appleton \& Lange, Norwalk, CT.

Hardt, M.E., Held, R., and Steinbach, M.J. 1971. Adaptation to displaced vision: A change in the central control of sensorimotor coordination. $J$. Exp. Psychol. 89: 229-239.

Harris, C.S. 1965. Perceptual adaptation to inverted, reversed, and displaced vision. Psychol. Rev. 72: 419-444.

Held, R., Efstathiou, A., and Greene, M. 1966. Adaptation to displaced and delayed visual feedback from the hand. J. Exp. Psychol. 72: 887-891.

Houk, J., and Henneman, E. 1967. Feedback control of skeletal muscles. Brain Res. 5: 433-451
Jakobson, L.S., and Goodale, M.A. 1989. Trajectories of reaches to prismatically-displaced targets: Evidence for "automatic" visuomotor recalibration. Exp. Brain Res. 78: 575-587.

Jeannerod, M. 1995. Corollary discharge in visuomotor coordination. In The handbook of brain theory and neural networks (ed. Michael A. Arbib), pp. 266-269. MIT Press, Cambridge, MA.

Kitazawa, S., Kohno, T., and Uka T.1995. Effects of delayed visual information on the rate and amount of prism adaptation in the human. J. Neurosci. 15: 7644-7652.

Kitazawa, S., Kimura, T., and Uka, T. 1997. Prism adaptation of reaching movements: Specificity for the velocity of reaching. J. Neurosci. 17: 1481-1492.

Kornheiser, A.S. 1976. Adaptation to laterally displaced vision: A review. Psychol. Bull. 83: 783-816.

Krakauer, J.W., Ghilardi, M.F., and Ghez, C. 1999. Independent learning of internal models for kinematic and dynamic control of reaching. Nat Neurosci. 2: (11) 1026-1031.

Martin, T.A., Keating, J.G., Goodkin, H.P., Bastian, A.J., and Thach, W.T. 1996. Throwing while looking through prisms. II. Specificity and storage of multiple gaze-throw calibrations. Brain 119: 1199-1211.

Shadmehr, R., and Mussa-Ivaldi, F.A. 1994. Adaptive representation of dynamics during learning of a motor task. J. Neurosci. 14: 3208-3224.

Sperry, R.W. 1950. Neural basis of the spontaneous optokinetic response produced by visual inversion. J. Comp. Physiol. Psychol. 43: 482-489.

von Holst, E., and Mittelstaedt, H. 1950. Das Reafferenzprinzip: Wechselwiskungen zwischen Zentralnervensystem und Peripherie. Naturwissenschaften 37: 464-476.

Welch, R.B. 1974. Research on adaptation to rearranged vision: 1966-1974. Perception 3: 367-392.

Welch, R.B., Choe, C.S., and Heinrich, D.R.1974. Evidence for a three-component model of prism adaptation. J. Exp. Psychol. 103: $700-705$.

Received November 8, 1999; accepted in revised form April 28, 2000. 


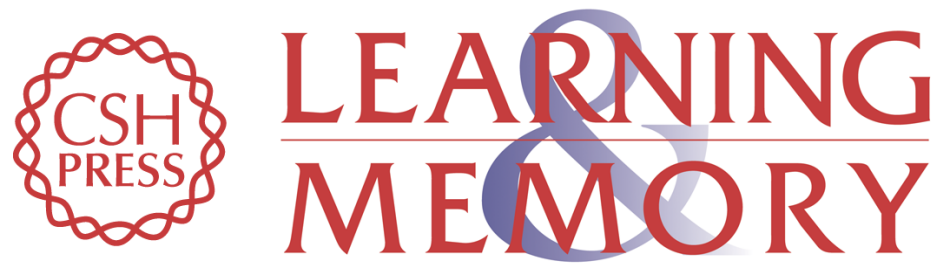

\section{Learning Motor Synergies Makes Use of Information on Muscular Load}

Juan Fernández-Ruiz, Cynthia Hall-Haro, Rosalinda Díaz, et al.

Learn. Mem. 2000, 7:

Access the most recent version at doi:10.1101//m.7.4.193

References This article cites 18 articles, 5 of which can be accessed free at:

http://learnmem.cshlp.org/content/7/4/193.full.html\#ref-list-1

License

Email Alerting Receive free email alerts when new articles cite this article - sign up in the box at the Service top right corner of the article or click here. 